\title{
Chronic hepatitis in man and in dog: a comparative update
}

\author{
Matteo Cerquetella ${ }^{1}$, Vittorio Giuliano ${ }^{2}$, Giacomo Rossi ${ }^{1}$, Simona Corsi $^{2}$, \\ Fulvio Laus ${ }^{1}$, Andrea Spaterna $^{1}$, Vincenzo Villanacci ${ }^{3}$ and Gabrio Bassotti ${ }^{2}$ \\ ${ }^{1}$ School of Medical Veterinary Sciences. University of Camerino. Matelica, Italy. ${ }^{2}$ Gastroenterology and Hepatology \\ Section. Department of Clinical and Experimental Medicine. Santa Maria della Misericordia Hospital. Perugia, Italy. \\ ${ }^{3}$ Second Pathology Section. Spedali Civili. Brescia, Italy
}

\begin{abstract}
Chronic hepatitis is a frequent pathologic condition encountered in both dogs and humans; however, in the latter etiologic factors are usually searched and found that allow targeted therapeutic approaches, whereas in dogs this is less frequent. This review will take into consideration chronic hepatitis in dogs, and discuss differences and similarities between the two species with respect to this disease.
\end{abstract}

Key words: Chronic hepatitis. Dog. Liver. Man.

Cerquetella M, Giuliano V, Rossi G, Corsi S, Laus F, Spaterna A, Villanacci V, Bassotti G. Chronic hepatitis in man and in dog: a comparative update. Rev Esp Enferm Dig 2012; 104: 203-209.

\section{INTRODUCTION}

Chronic hepatitis in dogs represents a pathologic condition that can be hypothesized clinically, in which symptoms may be almost nonspecific, laboratory test represent an important aid, but whose diagnosis can be confirmed and the disease classified only on an histopathological basis. Unlike humans, in dogs it is usually difficult to identify the underlying etiology of chronic hepatitis $(1,2)$; however, recent studies showed some similarities between liver reaction patterns and the pathogenesis of such disease in men and dogs $(3,4)$, and fibrosis is considered the common evolution in the two species (1).

Received: $17-10-11$

Accepted: 23-11-11.

Correspondence: Matteo Cerquetella, DVM. School of Veterinary Medical Sciences. University of Camerino. Via Circonvallazione, 93/95. 62024 Matelica (Macerata), Italy.

e-mail: matteo.cerquetella@unicam.it
Thus, the comparative study between man and dog in relation to chronic hepatitis may represent an interesting opportunity attempting to improve the clinical and therapeutic management in the two species.

\section{CHRONIC HEPATITIS IN DOGS}

\section{Etiological aspects}

The etiology of chronic hepatitis in dogs usually remains unknown, and for this reason many cases are classified as idiopathic. Such aspect is important for the negative implications on treatment strategies, that are mainly based on histopathology, and for the difficulties to formulate a prognosis $(1,2,5)$. Nevertheless, some causative agents are commonly associated to chronic hepatitis; for example, certain toxins (e.g., aflatoxin) or drugs (e.g., anti-convulsivants) have been associated to chronic hepatitis $(1,6)$, as well as infectious causes. For instance, although canine adenovirus type 1 is responsible of acute hepatitis, it has also been hypothesized its role in causing chronic hepatitis in partially immune dogs $(5,7,8)$; in addition, infections with leptospiral serogroups (e.g., L. interrogans grippotyphosa) $(8,9)$ other than those normally included in the vaccination schedule have been suggested as responsible for chronic hepatitis (10). Subclinical chronic hepatitis has also been associated with canine leishmaniosis (L. infantum) (11).

An important and well known cause of chronic hepatitis in the dog is that of the Bedlington terrier, in which a genetic mutation of copper transporting proteins (deletion of exon 2 in both alleles in the COMMD1 gene) causes accumulation of copper inside the hepatocytes, leading to inflammation and necrosis $(6,12-14)$. Copper accumulation, and chronic hepatitis, have also been suggested to be familial in West Highland White terrier, Skye terrier, Dalmatian and Labrador Retriever (6), and could be breedassociated in Doberman Pinscher (13), Anatolian Shepherd, Boxer, Norwich terrier, Wire Fox terrier, Airedale terrier, Bull terrier, Kerry Blue terrier, Cocker Spaniel, 
Golden Retriever, German Shepherd Dog, Collie, Pekingese, Keeshond, Bulldog, Schnauzer, Poodle, Dachshund, Old English Sheepdog and Samoyed (15). Although in these breeds it has not been convincingly demonstrated whether copper accumulation it is the cause of the disease rather than representing a concomitance with chronic hepatitis (1), a recent study on a group of Labrador Retrievers suggests a possible hereditary primary copper storage disorder in these animals (13). Further studies and the definition of shared criteria may help in the next future to define a copper-related chronic hepatitis as primary or secondary, as suggested by Spee et al., which proposed the histochemical detection of copper liver levels as an effective parameter for this purpose (14).

Furthermore, similarly to what happens in human medicine, in which inherited $\alpha-1$ antitrypsin deficiency may be associated with an accumulation of $\alpha-1$ antitrypsin in the liver, participating in the genesis of a chronic hepatitis, also in the dog (English cocker spaniels) elevated liver levels of that molecule have been associated to chronic hepatitis $(5,16,17)$.

\section{Clinical aspects}

In dogs, it has been suggested to consider a chronic hepatitis after finding four months of elevated serum alanine aminotransferase levels associated to characteristic histopathological changes (18). Clinically, the disorder is generally not characterized by specific signs (19); anorexia, vomiting, diarrhea, polydipsia and polyuria, fatigue but also jaundice, hepatic encephalopathy and ascites can be variably associated to the disease, depending on its severity $(1,19)$. In case of liver failure, coagulopathies may be present; cirrhosis can be associated with portal hypertension (6) and in dogs with chronic hepatitis, ascites has been considered a negative prognostic factor (20), as already known for humans (21).

Laboratory findings are usually unspecific, and even though they can suggest a problem involving the liver they do not highlight whether the condition is a chronic hepatitis or another problem affecting that organ (19).

Usually, in case of clinical and/or laboratory suspicion of hepatitis an ultrasonographic liver scan can be of diagnostic aid; the finding of an altered structure can be indicative of a liver problem (Figs. A and B) even though many cases of chronic hepatitis may not be associated with ultrasound abnormalities $(22,23)$; however, free fluid in the abdomen (ascites) can usually be easily identified $(22,23)$.

Histologically, a diagnosis of chronic hepatitis is done when apoptosis or necrosis of hepatocytes, regeneration and fibrosis, as well as mononuclear or mixed inflammatory infiltrate, is found. It is important to determine the activity (quantity and extent of the damage) and the stage (extent of fibrosis and presence of cirrhosis) of the disease $(5,6,19)$ (Figs. C and D). The essential diagnostic feature of canine chronic hepatitis is portal inflammation with

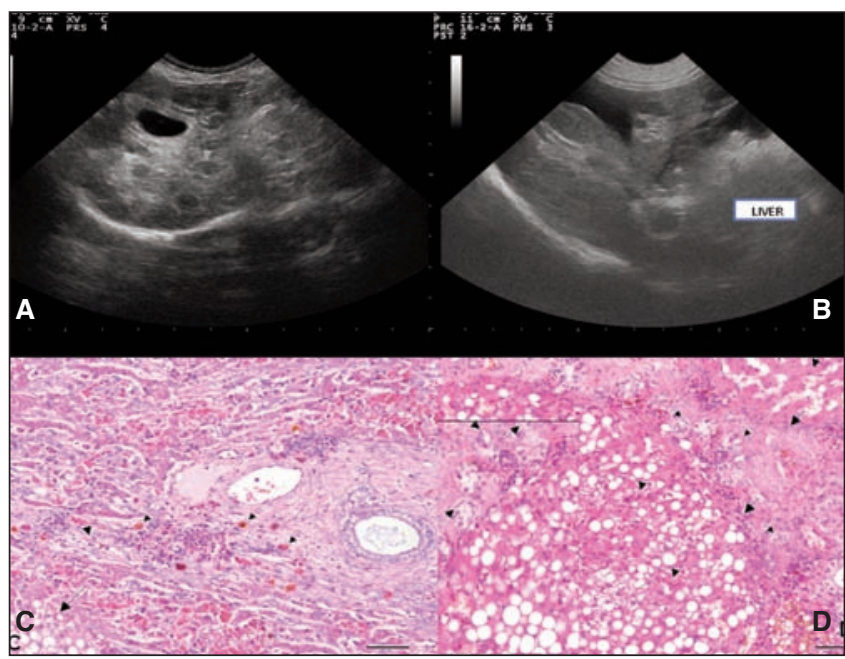

Fig. 1. A. Hepatic parenchymal heterogeneity with hypoechoic nodules alternated to hyperechoic areas. B. Altered structure of the hepatic parenchyma with hypoechoic areas and irregularly defined contours; anechoic peritoneal effusion is also present. C. Liver section characterized by a dissecting septum (arrowheads) consisting of fibrous connective tissue with formation of two sublobules. Fibrous connective tissue is prominent in the portal areas and thin bands tended to encircle small groups of hepatocytes. Note a generalized peri-centrolobular degeneration with ballooning or vacuolated hepatocytes (large arrow), and iron pigment deposition at the periphery of a sub-lobule (small arrows). A mild lymphoplasmacytic inflammatory infiltration in zone 1 is also present, associated with moderate reticulofibrosis. Case compatible with an histological diagnosis of chronic hepatitis. H\&E stain. Bar $=100 \mu \mathrm{m}$. D. Liver histology showing severe architectural disorder and abnormal nodules formation (arrowheads) associated with bands of connective tissue often extending from one central vein to another. A large regenerative nodule corresponds to the hypoechoic region seen on ultrasonography in $\mathrm{B}$, with collagen deposition along with a condensed reticulin network bordering the regenerative nodule (large arrows). This hepatic lobular collapse and fibrosis is mixed with severely vacuolated hepatocytes. Insert: high power view of the edge of the regenerative nodule, in which vacuolated hepatocytes (bottom), condensed reticulin network with bile ductules (small arrows), and the edge of the regenerative nodule (top) can be seen. H\&E stain. Bar = $100 \mu \mathrm{m}$.

absence of piecemeal necrosis (24). As described by some authors (25), fibrosing hepatitis results from a progression of piecemeal necrosis and consists in dissection of the lobular parenchyma by reticulin and fine collagen fibers with minimal portal inflammation (26). Two other conditions, histologically characterized by noninflammatory fibrosis, which varied from diffuse to zonal reticulofibrosis, have also been reported $(27,28)$ and named noninflammatory primary hepatic fibrosis (27) and idiopathic hepatic fibrosis (28). In the latter condition, the young age of the patients and overrepresentation of German shepherds suggested a genetic component (28). Fibrosis commonly occurs as a sequel to hepatic parenchymal damage and inflammation, often progresses to cirrhosis, and is associated with increases in interstitial collagen, basement membrane collagen (type IV), and proteoglycans (29). Disse's spaces contain increased amounts of 
laminin and fibronectin, as well as collagen (30,31) and hyperplasic Ito cell, whose role in hepatic fibrosis has been a topic of interest in the last decade. One of the likely initiating factors of fibrosis is changes involving cellular fibronectin, which seem to arise from activated lipocytes (32).

The evolution of a chronic hepatitis is represented by cirrhosis, characterized by diffuse fibrosis and rearrangement of liver architecture, with nodules and porto-central vascular anastomosis formation. Depending on nodules size, smaller or larger than $3 \mathrm{~mm}$, cirrhosis can be distinguished in micronodular or macronodular $(2,6)$.

Lobular dissecting hepatitis is a disease characterized by a rapid evolution and affecting young dogs, in which fibroblasts and extracellular matrix modify the lobular structure of the liver laying between hepatocytes (6).

Eosinophilic hepatitis is a rare condition associated to allergic conditions, hypereosinophilic syndromes, parasites, or to drug-related disease, consisting in a non-specific reactive hepatitis characterized by a scattered eosinophilic infiltrate (6).

The presence of an autoimmune hepatitis is still uncertain in the dog because, even though in some cases of chronic hepatitis it has been shown a lymphocytic (CD3+ lymphocytes) infiltrate, it is not clear if this represents a primary or a secondary finding (1), although suggestive of an immune-mediated process (9). The correlation between chronic hepatitis and an immune-mediated process has also been suggested in a study in which in the liver of a dog affected by chronic hepatitis was found an increased ratio of CD4+/CD8+ lymphocytes (33), and in a study in dogs with chronic hepatitis showing proliferation of peripheral blood mononuclear cells in response to liver membrane antigens (34). Furthermore, a study performed on Dobermans affected by hepatitis at different clinical stages, showed upregulation of MHC class II antigens in hepatocytes, leading the authors to hypothesize an autoimmune ground (35), similarly to another study in which for Doberman hepatitis, showing an association between the disease and the homozygous haplotype DLADRB1*00601/DQA1*00401/DQB $1 * 01303$, it has been hypothesized an immune origin (36).

\section{CHRONIC HEPATITIS IN MEN}

- Chronic hepatitis in humans usually occurs without symptoms, and it is often diagnosed after blood tests performed for screening purposes. Differently than in dogs, several etiologic causes, such as alcohol, viruses, metabolic, autoimmune and hereditary factors, have been identified in men, even though the pathogenic mechanisms are not still completely clarified. The diagnosis of chronic hepatitis is based on patient history, serological tests, clinical context (i.e., the presence of metabolic syndrome, neurological symptoms, organ dysfunction). In variable percentage, chronic hepatitis may evolve to cir- rhosis, the diagnosis being sometimes disclosed by the occurrence of acute complications such as variceal bleeding, encephalopathy, ascites, etc. Screening ultrasound in asymptomatic patients may reveal signs of unknown liver disease and incidental hepatocellular carcinoma (HCC).

- Alcoholic hepatitis. Ethanol abuse is a relevant cause of liver damage. The spectrum of disease ranges from fatty liver to hepatic inflammation, necrosis, progressive fibrosis and HCC. The pathogenesis of liver damage involves abnormalities of energy metabolism, oxidative stress and immunologic mechanism. Chronic alcohol use depresses the activity of all mitochondrial complexes, and causes hypoxia due to liver cells increased requirement deriving from ethanol metabolism. Alcohol also leads to increased liver oxidative stress trough generation of acetaldehyde; this molecule can form hybrid adducts with reactive residues acting on proteins or small molecules, mediating lipid peroxidation and nucleic acid oxidation (37). Ethanol intake increases intestinal permeability to a variety of substances, including bacterial endotoxins, that trigger proinflammatory cytokine release, namely TNF-alfa, TGF-beta, IL-6 (38). In addition, concomitant obesity and viral and iron overload aggravate the severity and the progression of alcoholic liver disease.

- Chronic viral hepatitis. In humans, five known hepatotropic viruses, the hepatitis A, B, C, D, E viruses (HAV, $\mathrm{HBV}, \mathrm{HCV}, \mathrm{HDV}, \mathrm{HEV}$ ) are etiologically linked to viral hepatitis. Of these, HEV, HBV, HDV and HCV are parenterally transmitted and can cause acute infection with a high propensity to become chronic with long term sequelae, such as cirrhosis and liver cancer. Viral contact generally involves transfusion of blood products, injection with contaminated needles, unprotected sexual activity, and vertical transmission.

These viruses are not cytopathic and hepatocyte necrosis is probably a consequence of cytotoxic T-cell response directed against membrane-bound viral antigens on hepatocytes.

Despite significant overlap in clinical manifestations, hepatitis viruses differ widely in their morphology, genomic organization and modes of replication.

HBV genome is a partial double-stranded circular DNA with sequences encoding the viral core ( $\mathrm{HBcAg}$ ), envelope (HBsAg), polymerase $(\mathrm{P})$ and $\mathrm{HBx}$ proteins. The viral genomic DNA is transferred to the nucleus, where the partially double stranded DNA is circular-closed (cccDNA), and serves as a template for transcription of four viral mRNAs with the help of host RNA polymerase. The largest transcripted mRNA, also called pregenomic RNA, is used as a template to make new copies of the genome and to make the capsid protein and the viral DNA polymerase; pregenomic RNA is then transported back to the cytoplasm where the viral DNA polymerase makes a DNA copy. The viral particles begin to assemble at the hepatocyte surface and are then released. Sera of $\mathrm{HBV}$ 
patients carry large number of empty particles composed of envelope proteins, and the infected sera may contain also Dane particles, which represent the intact virus. The most effective measure of prevention is routine immunization for infants and high-risk individuals. Passive immunization is reserved to infants born from $\mathrm{HBsAg}$ carrier mothers. Therapy of HBV chronic hepatitis includes recombinant and pegylated IFN-alfa and nucleoside/nucleotide analogues (entecavir, telbivudine, adefovir, lamivudine, tenofovir). The goal of therapy resides on virus eradication and the delay or the interruption of disease progression.

HDV virus is considered a sub-viral satellite because it can propagate only in HBsAg carrier individuals. Virus transmission can occur either with simultaneous infection with HBV (co-infection) or with HBsAg carriers superinfection. These patients progress more rapidly toward cirrhosis and may even develop HCC. In chronic infection, HDV RNA, HDVAg, anti HDV antibodies persist and allow diagnosis. There is no specific therapy for HDV hepatitis, mainly based on interferon therapy. As HDV needs HBV for its propagation, though in absence of an available vaccine, HDV infection can be prevented by hepatitis B vaccination.

Hepatitis $\mathrm{C}$ virus causes a long-term chronic infection in $70-80 \%$ of infected patients, it is a member of flaviviridae family with a genome consisting of a single strained RNA molecule. HCV enters target cells by a highly coordinated process involving components of the virus particle and numerous cellular factors. Intriguingly, HCV entry is restricted only to human and chimpanzee cells due to the lack of specific receptors in other animals. Less than one third of patients spontaneously clear a primary $\mathrm{HCV}$ infection. It has been shown that CD4+ $\mathrm{T}$ cell response appears to be critical to coordinate effective immunity (39). HCV RNA translation is unique and basically different from host cell RNA translation; thus, it may be exploited to develop selective antiviral agents. Diagnosis of chronic viral infections is made by monitoring biochemical, virological and histological parameters. Anti-HCV antibodies and qualitative and quantitative molecular methods (real time PCR) are used to assess HCV infection; detection of HCV RNA in blood confirms the presence of virus because anti-HCV antibodies can only indicate the exposure to virus. At present no vaccine candidate is available. A majority of antiviral research at the moment is focused on protease and polymerase inhibitors $(40,41)$.

- Metabolic liver disease. Non alcoholic steatohepatitis (NASH) is a liver disease characterized by the presence of fatty infiltration with inflammation that resembles alcoholic steatohepatitis in non-drinkers (42). The term non-alcoholic fatty liver disease (NAFLD) has been adopted to cover the full spectrum of metabolic fatty liver disease, particularly when histology is undefined (43). Fortunately, only a minority of patients with NAFLD progresses to more advanced disease characterised by inflam- mation, fibrosis, cirrhosis and HCC. Population studies show that NAFLD is strongly associated with obesity, insulin resistance/type II diabetes mellitus, and dyslipidemia, and it is considered the hepatic manifestation of the metabolic syndrome (44). One large European study found NAFLD present in $94 \%$ of obese patients (BMI > $\left.30 \mathrm{~kg} / \mathrm{m}^{2}\right), 67 \%$ of overweight patients $\left(\mathrm{BMI}>25 \mathrm{~kg} / \mathrm{m}^{2}\right)$, and $25 \%$ of normal weight patients (45). Insulin resistance is considered the initiating event in NAFLD/NASH and promoting hepatic free fatty acid (FFA) flux provides the appropriate substrate for NAFLD/NASH development. In addition, direct hepatocyte lipotoxicity, hepatocellular oxidative stress secondary to free radicals produced during $\beta$ - and $\omega$-FFA oxidation, endotoxin/TLR4 induced Kupffer cell cytokine release, lead to stellate cell activation and hepatic fibrosis. Clinicians' attention is focused on insulin "sensitizing" drugs such as glitazones, which can be used to treat the liver disorder, but lifestyle measures, diet and increasing physical activity through aerobic exercise have been shown to be crucial for treatment of this condition (46).

- Hereditary diseases. Metal accumulation is the main cause of adult hereditary chronic hepatitis, causing systemic diseases that involve many organs with consequent clinical syndromes. "Bronze diabetes" or hereditary hemochromatosis is a well defined iron load syndrome, characterized by the toxic accumulation of iron in liver and endocrine glands. Most cases of hemochromatosis arise from genetic alterations, including $H F E$ (mutated in more than $80 \%$ of cases of hemochromatosis), $T f R 2$, and $H J V$. These genes regulate hepcidin synthesis, a protein involved in iron homeostasis. Increased saturation of transferrin (which transports iron to cells such as erythroid precursors in the bone marrow) and iron accumulation in the parenchymal cells of various organs (liver, gonads, pancreas, heart, and skin), is related to the deficient or reduced synthesis of hepcidin, which down-regulates the entry of iron into the bloodstream (47). Wilson's disease or hepatolenticular degeneration is a rare autosomal recessive disorder caused by mutation in the ATP7B gene, with resultant impairment of biliary excretion of copper. Metal accumulation, firstly in the liver but ultimately in the brain and other tissues, produces protean clinical manifestations that may include hepatic, neurological, psychiatric and ophthalmological alterations. Free copper is extremely toxic and can produce irreversible cellular damage. Both the ATP7B protein and ceruloplasmin are involved with copper transport; the ATP7B protein mediates the incorporation of six copper molecules into apoceruloplasmin, forming ceruloplasmin. ATP7B transports excess copper across the hepatocyte apical membrane into bile canalicula for subsequent biliary excretion. Mutation in the ATP7B gene occurring in Wilson's disease, results in defective ATP7B protein that cannot perform these functions (48). The consequent progressive copper accumulation ultimately compromise hepatic function; when the hepatic storage capacity is 
exceeded, unbound copper spills out of the liver and is deposited in other organs and tissues, where it also provokes damage and dysfunction. Chelating agents such as penicillamine, promoting copper excretion, represent the principal therapeutic weapon for Wilson's disease; zinc acetate is commonly used in maintaining therapy after copper depletion and has been shown to be effective in neurological syndrome and in children (49).

- Autoimmune disorders. Autoimmune hepatitis $(\mathrm{AIH})$ is a chronic inflammatory disease of unknown etiology characterized by the presence of circulating autoantibodies, hypergammaglobulinemia ( $\mathrm{IgG})$, necroinflammatory changes on hepatic histology and a dramatic response to immunosuppressive drugs (50). Although more frequent in young women (at least $70 \%$ of cases and $50 \%$ are younger than 40 years), AIH can affect children and adults of all ages and ethnicities; $25-34 \%$ of patients present with asymptomatic liver test abnormalities, and $40 \%$ of them may have an acute onset, but the presentation of severe fulminant hepatic failure is rare. In children or elderly patients the disease commonly presents with liver cirrhosis. No single clinical or biochemical test proves the presence of AIH and autoantibodies are nonspecific; an exception may be the presence of soluble liver antigen/liver -pancreas (SLA/LP) autoantibodies (present in about 20\% of patients). However, the presence of autoantibodies in the serum is of diagnostic importance and helps in categorizing the various subtypes of AIH. Type $1 \mathrm{AIH}$ is characterized by the presence of antinuclear antibody (ANA), anti smooth muscle antibody (SMA) or both, and represents $80 \%$ of AIH cases; about $25 \%$ have cirrhosis at the presentation and the association with other autoimmune disease is common. Type $2 \mathrm{AIH}$ is characterized by the presence of anti liver kidney microsomal (LKM)1 and/or LKM3 and/or anti liver cytosol $(\mathrm{LCl})$ antibodies. Most patients are children, acute severe presentation can occur, and progression to cirrhosis commonly ensues. As a rule, autoantibodies should not be used to monitor therapy, predict AIH activity or outcome. Anti-nuclear antibodies (ANA) are encountered in about $80 \%$ of $\mathrm{AIH}$ patients in titers greater than 1:40 and anti-smooth muscle antibodies (ASMA) in titers greater than 1:40 are seen in approximately $70 \%$. Both ANA and ASMA, in low titers, may be seen in other chronic liver diseases such as HCV, Wilson disease, alcohol-related liver disease, or NASH. Although the exact pathogenesis is still unknown, AIH is thought to be caused by environmental triggers (viruses or drugs) which cause the failure of immune tolerance in a genetically susceptible host. The underlying genetic predisposition has been suggested because patients are predominantly female and the association of the disease with certain human leucocytes antigens (HLAs); in particular, HLA B8, DR3 and DR4 are found to be significantly frequent in different populations with AIH. The histological hallmark of AIH is lymphoplasmacytic periportal infiltrate invading the limiting plate, also called piecemeal necrosis or "interface hepatitis", that eventually progresses to lobular hepatitis. There is often abundance of plasma cells and eosinophils and a lobular, or panacinar hepatitis is frequently observed. Fibrosis is present in all but the mildest forms of $\mathrm{AIH}$, and it causes distortion of the hepatic lobule and the appearance of regenerative nodules, resulting in cirrhosis. Immunosuppressive therapy with corticosteroids usually in combination with azathioprine is considered the gold standard to induce and maintain remission (51); alternative treatments employ budesonide and mycophenolate mofetil.

\section{CONCLUSIONS}

Primary hepatitis is a frequently encountered liver disease in the dog, and represents $0.5-1 \%$ of the case load (depending on the study); about two thirds of these patients are affected by chronic hepatitis $(2,18,23)$. Presently, many clinical cases of chronic hepatitis in dogs are classified as idiopathic, while in humans an etiologic diagnosis is usually sought to start a targeted therapy $(1,2)$. However, by considering some similarities in liver reaction patterns in the two species comparative studies could yield useful data to increase bilaterally the knowledge on the disease $(3,4)$. This concept is strengthened by the fact that similarities in the hepatic fibrotic process (transforming growth factor $\beta-1$ (TGF $\beta-1$ ) expression and Smad 2/3 phosphorylation) in man and dog have been demonstrated (3). TGF $\beta$ is produced by perisinusoidally located hepatic stellate cells directly involved in fibrogenesis, by stimulation release of collagens and glycoaminoglycans and of inhibitors of metalloproteinases (2). In addition, an immunohistochemical study conducted on cytokeratin-7 (CK7), human hepatocyte marker 1 (Hep Par 1), multidrug resistance-associated protein-2 (MRP2), and breast cancer resistance protein (BCRP) showed several similarities in the two species, in both normal liver samples and pathological ones (acute and chronic liver disease) (4).

In the dog, $\alpha$-smooth muscle actin positive cells, similar to stellate cells and myofibroblasts, seem to be correlated with inflammation and fibrosis, as it has been shown in man $(1,9)$. The role of these cells is important because they could represent the base for strategies directed against fibrosis (1).

Copper storage disorders are known both in man and in dog, but even though there are similarities between human Wilson's disease and the Bedlington terrier one (12), there are also differences, owing to the fact that in man the accumulation is present not only in the liver, as it happens in Bedlington terrier, but also in other organs like the eyes (1) or the brain (52) and that different genes are involved in Wilson's disease and in Bedlington terrier copper storage disorder (15).

Further studies are needed to widen our knowledge on autoimmune hepatitis in the dog. 


\section{ACKNOWLEDGMENTS}

We are warmly indebted to Antony Buttaro, Jr for reviewing the English form of the manuscript.

\section{REFERENCES}

1. Watson PJ. Chronic hepatitis in dogs: a review of current understanding of aetiology, progression, and treatment. Vet J 2004;167:228-41.

2. Favier RP. Idiopathic hepatitis and cirrhosis in dogs. Vet Clin Small Anim 2009;39:481-8.

3. Spee B, Arends B, van den Ingh TSGAM, Brinkhof B, Nederbragt H, Ijzer J, et al. Transforming growth factor -1 signalling in canine hepatic diseases: new models for human fibrotic liver pathologies. Liver International 2006;26:716-25.

4. Ijzer J, Schotanus BA, Vander Borght S, Roskams TAD, Kisjes R, Penning LC, et al. Characterization of the hepatic progenitor cell compartment in normal liver and in hepatitis: an immunohistochemical comparison between dog and man. Vet J 2010;184:308-14.

5. Boomkens SY, Penning LC, Egberink HF, van den Ingh TSGAM, Rothuizen J. Hepatitis with special reference to dogs. A review on the pathogenesis and infectious etiologies, including unpublished results of recent own studies. Vet Q 2004;26:107-14.

6. van den Ingh TSGAM, Van Winkle T, Cullen JM, Charles JA, Desmet VJ. Morphological classification of parenchymal disorders of the canine and feline liver -2. Hepatocellular death, hepatitis and cirrhosis. In: WSAVA Standards for clinical and histological diagnosis of canine and feline liver diseases. Saunders Elsevier; 2006. p. 85-102.

7. Gocke DJ, Preisig R, Morris TQ, McKay DG, Bradley SE. Experimental viral hepatitis in the dog: production of persistent disease in partially immune animals. J Clin Invest 1967;46:1506-17.

8. Chouinard L, Martineau D, Forget C, Girard C. Use of polymerase chain reaction and immunohistochemistry for detection of canine adenovirus type 1 in formalin-fixed, paraffin-embedded liver of dogs with chronic hepatitis or cirrhosis. J Vet Diagn Invest 1998;10:320-5.

9. Boisclair J, Doré M, Beauchamp G, Chouinard L, Girard C. Characterization of the inflammatory infiltrate in canine chronic hepatitis. Vet Pathol 2001;38:628-35.

10. Adamus C, Buggin-Daubié M, Izembart A, Sonrier-Pierre C, Guigand L, Masson MT, Chronic hepatitis associated with leptospiral infection in vaccinated beagles. J Comp Path 1997;117:311-28.

11. Rallis T, Day MJ, Saridomichelakis MN, Adamama-Moraitou KK, Papazoglou L, Fytianou A, et al. Chronic hepatitis associated with canine leishmaniosis (Leishmania infantum): a clinicopathological study of 26 cases. J Comp Path 2005;132:145-52.

12. van de Sluis B, Rothuizen J, Pearson PL, van Oost BA, Wijmenga. Identification of a new copper metabolism gene by positional cloning in a purebred dog population. Hum Mol Gen 2002;11:165-73.

13. Hoffmann G, van den Ingh TSGAM, Bode P, Rothuizen J. Copperassociated chronic hepatitis in labrador retrievers. J Vet Intern Med 2006;20:856-61.

14. Spee B, Arends B, van den Ingh TSGAM, Penning LC, Rothuizen J. Copper metabolism and oxidative stress in chronic inflammatory and cholestatic liver diseases in dogs. J Vet Intern Med 2006;20:1085-92.

15. Willard MD. Inflammatory canine hepatic disease. In: Ettinger SJ, Feldman EC, editors. Textbook of Veterinary Internal Medicine. 7th ed. St. Louis: Saunders; 2010. p. 1637-42.

16. Sevelius E, Andersson M, Jönsson L. Hepatic accumulation of alpha1-antitrypsin in chronic liver disease in the dog. J Comp Pathol 1994;111:401-12.

17. Vatne M, Andersson M, Sevelius E, Jönsson L. Immunohistochemical investigation of four glycoproteins in the hepatocytes of dogs with chronic liver disease. Eur J Vet Pathol 2001;7:51-9.

18. Fuentealba C, Guest S, Haywood S, Horney B. Chronic hepatitis: a retrospective study in 34 dogs. Can Vet J 1997;38:365-73.

19. Sterczer A, Gaál T, Perge E, Rothuizen J. Chronic hepatitis in the dog: a review. Vet Q 2001;23:148-52.

20. Raffan E, McCallum A, Scase TJ, Watson PJ. Ascites is a negative prognostic indicator in chronic hepatitis in dogs. J Vet Intern Med 2009;23:63-6
21. Rahimi RS, Rockey DC. Complications and outcomes in chronic liver disease. Curr Opin Gastroenterol 2011;27:204-9.

22. Watson P. Diseases of the liver. In: Hall EJ, Simpson JW, Williams DA, editors. In: BSAVA. Manual of canine and feline gastroenterology. 2nd ed. Gloucester; 2005. p. 240-68.

23. Poldervaart JH, Favier RP, Penning LC, Van den Ingh TSGAM, Rothuizen J. Primary hepatitis in dogs: a retrospective review (2002-2006). J Vet Intern Med 2009;23:72-80.

24. Scheuer PJ. Liver biopsy interpretation. $4^{\text {th }}$ ed. London: Bailliere Tindall; 1988.

25. Thornburg LP, Childs A, Toomey AA, Roudebush P. Postnecrotic canine cirrhosis I. Clinicopathologic features. Vet Clin N Am-Small 1983;78:43-50

26. Bennett AM, Davies JD, Gaskell CJ, Lucke VM. Lobular dissecting hepatitis in the dog. Vet Pathol 1983;20:179-88.

27. van den Ingh TSG, Rothuizen J. Hepatoportal fibrosis in three young dogs. Vet Rec 1982;110:575-7.

28. Rutgers HC, Haywood S, Kelly DF. Idiopathic hepatic fibrosis in 15 dogs. Vet Rec 1993;133:115-8.

29. Bissell DM. Cell-matrix interaction and hepatic fibrosis. In: Popper $\mathrm{H}$, Schaffner F, editors. Progress in liver diseases. Vol 9. Philadelphia: WB Saunders; 1990. p.143-55.

30. Hahn E, Wick G, Pencev D, Timpl R. Distribution of basement membrane proteins in normal and fibrotic human liver: collagen type IV, laminin, and fibronectin. Gut 1980;21:63-71.

31. Clement B, Rescan P-Y, Baffet G, Loréal O, Lehry D, Campion JP, et al. Hepatocytes may produce laminin in fibrotic liver and in primary culture. Hepatology 1988;8:794-803.

32. Ramadori G, Rieder H, Knittel TH, Dienes HP, Meyer zum Büschenfelde C. Fat storing cells (FSC) of rat liver synthesize and secrete fibronectin. J Hepatol 1987;4:190-7.

33. Sakai M, Otani I, Ishigaki K, Edamura K, Takahashi T, Koie H, et al Phenotypic analysis of hepatic T lymphocytes in a dog with chronic hepatitis. J Vet Med Sci 2006;68:1219-21.

34. Poitout F, Weiss DJ, Armstrong PJ. Cell-mediated immune response to liver membrane protein in canine chronic hepatitis. Vet Immunol Immunopathol 1997:57:169-78.

35. Speeti M, Ståhls A, Meri S, Westermarck E. Upregulation of major histocompatibility complex class II antigens in hepatocytes in Doberman hepatitis. Vet Immunol Immunopathol 2003;96: 1-12.

36. Dyggve H, Kennedy L J, Meri S, Spillmann T, Lohi H, Speeti M. Association of Doberman hepatitis to canine major histocompatibility complex II. Tissue Antigens 2010;77:30-5.

37. Lieber CS. Biochemical factors in alcoholic liver disease. Semin Liver Dis 1993; 13:136-53

38. Nolan JP. Intestinal endotoxins as mediators of hepatic injury - an idea whose time has come again. Hepatology 1989;10:887-91.

39. Saito T, Owen DM, Jiang F, Marcotrigiano J, Gale M. Innate immunity induced by composition-dependent RIG-I recognition of hepatitis C virus RNA. Nature 2008;454:523-7.

40. Fried MW Viral factors affecting the outcome of therapy for chronic hepatitis C. Rev Gastroenterol Disord 2004;4:S8-S13.

41. Chen KX, Njoroge FG. A review of HCV protease inhibitors. Curr Opin Investigat Drugs 2009;10:821-37.

42. Ludwig J, Viggiano T, McGill D, Ott B. Nonalcoholic steatohepatitis. Mayo clinic experiences with a hitherto unnamed disease. Mayo Clin Proc 1980;55:434-8.

43. Angulo P. Nonalcoholic fatty liver disease. N Engl J Med 2002; $16 ; 1221-31$

44. Marchesini G, Brizi M, Morselli-Labate AM, Bianchi G, Bugianesi E, McCullough AJ, et al. Association of nonalcoholic fatty liver disease with insulin resistance. Am J Med 1999;107: $450-5$.

45. Argo CK, Caldwell SK. Epidemiology and natural history of non-alcoholic steatohepatitis, Clin Liver Dis 2009;13:511-31.

46. Dowman JK, Armstrong MJ, Tomlinson JW, Newsome PN. Current therapeutic strategies in non-alcoholic fatty liver disease. Diabetes Obes Metab 2011;13:692-702.

47. Ganz T. Hepcidin, a key regulator of iron metabolism and mediator of anemia of inflammation. Blood 2003;102:783-8.

48. Ala A, Schilsky M. Genetic modifiers of liver injury in hereditary liver disease. Semin Liver Dis 2011;31:208-14. 
49. Linn FH, Houwen RH, van Hattum J, van der Kleij S, van Erpecum KJ. Long-term exclusive zinc monotherapy in symptomatic Wilson disease: experience in 17 patients. Hepatology 2009;50:144252.

50. Mieli-Vergani G, Vergani D. Autoimmune hepatitis. Nat Rev Gastroenterol Hepatol 2011;8:320-9.
51. Makol A, Watt KD, Chowdhary VR. Autoimmune hepatitis: a review of current diagnosis and treatment. Hepat Res Treat 2011;2011:390916.

52. Richter KP: Diseases of the Liver and Hepatobiliary System. In: Tams TR, editor. Handbook of small animal gastroenterology. 2nd ed. St. Louis: Saunders; 2003. p. 286-352. 